MOTION 



\title{
AMBIGUITY IN RECONSTRUCTION FROM IMAGE CORRESPONDENCES
}

\author{
Stephen J. Maybank \\ Long Range Laboratory, GEC Hirst Research Centre, East Lane, \\ Wembley, Middlesex HA9 7PP, UK
}

\section{Introduction}

The possibility of reconstructing the shape of the environment from the correspondences between two images first arose during the 19th century with the invention of photography. The methods developed for exploiting this possibility were based on projective geometry since projection provides a good model for image formation. At first the shape of the environment was reconstructed by linear methods [16], which did not make full use of the rigidity of the environment. Later methods incorporated the rigidity constraint $[3,7]$, thus allowing reconstruction with fewer image correspondences, but at the cost of greatly increasing the complexity of the reconstruction algorithm.

More recently the reconstruction problem has been transformed by the advent of electronic cameras and computers. Large numbers of images are obtained by an electronic camera in a short space of time, the image correspondences are found automatically, and reconstruction is carried out by a computer algorithm. In this way a robot or an automatic vehicle can obtain useful information by passive means well suited to a wide variety of environments. The modern approach to reconstruction is based on Euclidean geometry and the vector calculus, rather than on projective geometry $[3,6,8]$. A very large number of algorithms for reconstruction have been published recently. An example of a linear algorithm may be found in [8], and examples of non-linear algorithms may be found in [6], together with further references to the literature.

In some cases reconstruction from image correspondences is ambiguous, in that two essentially different surfaces in space are obtained. Ambiguity has been studied previously using both the older projective geometric approach $[3,4,17]$ and the newer Euclidean approach $[3,5,6,9]$. In this paper projective geometric and Euclidean techniques are used together to prove that ambiguous surfaces are invariant under a rotation through $180^{\circ}$. The rotation interchanges the two possible positions for the optical centre of the camera taking the second image. In consequence, a cubic polynomial constraint on ambiguous surfaces is obtained, which forms the basis of a new proof of Demazure's result [1] that there are in general exactly ten camera displacements compatible with five given image correspondences.

The invariance of an ambiguous surface under a rotation through $180^{\circ}$ was first discovered in photogrammetry [4]. The cubic polynomial constraint on ambiguous surfaces is new. An extensive discussion of the projective geometric approach to ambiguity including alternative proofs of some of the results given here will appear in [14].

\subsection{Notation}

The necessary background in projective geometry is given in [15]. Euclidean three dimensional space $\mathbf{R}^{3}$ is regarded as a subset of projective space $\mathbf{P}^{3}$. Points of $\mathbf{R}^{3}$ are denoted by $\left[x_{1}, x_{2}, x_{3}\right]$, and points of $\mathbf{P}^{3}$ are denoted by $\left(x_{1}, x_{2}, x_{3}, x_{4}\right)$. Two points of $\mathbf{P}^{3},\left(x_{1}, x_{2}, x_{3}, x_{4}\right)$ and $\left(x_{1}^{\prime}, x_{2}^{\prime}, x_{3}^{\prime}, x_{4}^{\prime}\right)$ are the same if and only if there exists a non-zero scalar $\lambda$ such that $x_{i}=\lambda x_{i}^{\prime}$ for $1 \leq i \leq 4$. Thus, in $\mathbf{P}^{3}$,

$$
\left(x_{1}, x_{2}, x_{3}, x_{4}\right)=\lambda\left(x_{1}, x_{2}, x_{3}, x_{4}\right)
$$

It follows from (1) that the zeros of a polynomial equation $f(x)=0$ are well defined in $P^{3}$ if and only $f(\mathbf{x})$ is homogeneous in the coordinates $x_{i}$ of $\mathbf{x}$. 
The embedding $\mathbf{R}^{3} \subset \mathbf{P}^{3}$ is the usual one $\left[x_{1}, x_{2}, x_{3}\right] \mapsto\left(x_{1}, x_{2}, x_{3}, 1\right)$. The same symbol $\mathrm{x}$ is used to denote both $\left[x_{1}, x_{2}, x_{3}\right]$ and $\left(x_{1}, x_{2}, x_{3}, 1\right)$, depending on context. The set $\mathbf{P}^{3} \backslash \mathbf{R}^{3}$ is the plane at infinity, $\Pi_{\infty}$. The equation of $\Pi_{\infty}$ is $x_{4}=0$. The coordinates $x_{i}$ of $\mathbf{R}^{3}$ (and $\mathbf{P}^{3}$ ) are chosen to be rectangular. In this case the $x_{i}$ are referred to as Cartesian coordinates. The origin $(0,0,0,1)$ of Cartesian coordinates is denoted by 0 .

Points of $\mathbf{P}^{3}$ are typically denoted by $\mathbf{o}, \mathbf{a}, \mathbf{b}, \mathbf{x}, \mathbf{y}$, and lines of $\mathbf{P}^{3}$ are denoted by $g, h, k$. The line joining two distinct points $\mathbf{a}, \mathbf{b}$ of $P^{3}$ is $\langle\mathbf{a}, \mathbf{b}\rangle$, and similarly, the plane containing the line $g$ and the point a not on $g$ is $\langle g, \mathbf{a}\rangle$. This notation is extended to arbitrary numbers of lines and points. For example, $\langle g, h, \mathbf{a}\rangle$ is the smallest subspace of $\mathbf{P}^{3}$ containing the lines $g, h$ and the point a. Planes in $P^{3}$ are typically denoted by $\Pi, \Phi, \Xi$. Quadric surfaces in $P^{3}$ are typically denoted by $\psi$.

Each point $\mathbf{x}$ of $\mathbf{R}^{3}$ defines a vector, namely, the line segment from the origin $o$ to $\mathbf{x}$. This vector is denoted by the same symbol $\mathbf{x}$ as the point $\mathbf{x}$. The dot product $\mathbf{x} . \mathbf{y}$ and the vector product $\mathbf{x} \times \mathbf{y}$ of vectors $\mathrm{x}, \mathrm{y}$ are formed in the usual way. Each non-zero vector $\mathrm{x}=\left(x_{1}, x_{2}, x_{3}, x_{4}\right)$ corresponds to a unique point $\left(x_{1}, x_{2}, x_{3}, 0\right)$ of $\Pi_{\infty}$, which can be thought of as the direction of $\mathbf{x}$. If $\mathbf{x}, \mathbf{y}$ are points of $\Pi_{\infty}$, then $\mathbf{x} \times \mathbf{y}$ is defined as the unique point of $\Pi_{\infty}$ corresponding to the direction orthogonal to the directions of $x$ and $y$.

The tensor product of two vectors $\mathbf{x}, \mathbf{y}$ is denoted by $\mathbf{x} \otimes \mathbf{y}$. In the applications of the tensor product made in this paper $x$ and $y$ are points of $P^{3}$ contained in $\Pi_{\infty}$. Then $\mathbf{x} \otimes \mathbf{y}$ is the $3 \times 3$ matrix with $i, j$ th entry equal to $x_{i} y_{j}$ for $1 \leq i, j \leq 3$. The coordinates of $\mathrm{x}$ and $\mathrm{y}$ are only defined up to a non-zero scalar multiple, thus $\mathbf{x} \otimes \mathbf{y}$ is also only defined up to a non-zero scalar multiple.

Invertible linear transformations, or collineations, are typically denoted by $\omega, r$. The value of $\omega$ at a point $\mathbf{x}$ is $\omega \mathbf{x}$ (without brackets). If $S$ is a set of points, for example a line, or a plane, then $\omega(S)$ (with brackets) is the set of $\omega \mathbf{x}$ as $\mathbf{x}$ ranges over $S$. If $\omega(S) \subset S$ then $S$ is said to be invariant under $\omega$.

\section{Reconstruction from image correspondences}

The usual formulation of the reconstruction problem is employed [10]. Two images of the same set of scene points $p_{i}$ are taken from distinct projection points, $o$ and $a$. It is assumed that the $p_{i}$ are fixed rigidly in space with respect to $o$ and $a$. The point $o$ is referred to as the optical centre of the first camera, and $a$ is referred to as the optical centre of the second camera. The imaging surface of each camera is the unit sphere with centre at the optical centre of the camera. The image is formed by polar projection towards the optical centre. Each scene point $\mathbf{p}_{i}$ gives rise to points $\mathbf{q}_{i}, \mathbf{q}_{i}^{\prime}$ in the first and second images respectively. The correspondence between $\mathbf{q}_{i}$ and $\mathbf{q}_{i}^{\prime}$ is denoted by $\mathbf{q}_{i} \leftrightarrow \mathbf{q}_{i}^{\prime}$.

In practice, the camera projection is more complicated than polar projection onto the unit sphere. This discrepancy between theory and experiment is overcome by calibrating the camera. The acquired image is transformed in order to obtain the image that would have arisen from polar projection.

\subsection{The Euclidean approach}

In the Euclidean approach to reconstruction each camera has associated to it a coordinate frame in which the positions of the image points are measured. The displacement of the second camera with respect to the first is specified by giving both the translation from the first optical centre $o$ to the second optical centre $\mathbf{a}$, and the rotation $R$ needed to bring the two camera coordinate frames into alignment after the translation. It is assumed that $\operatorname{det}(R)=1$, thus excluding the possibility that the two coordinate frames differ by a reflection. When specifying the relative position of the cameras by a pair $\{R, a\}$ it is assumed that Cartesian coordinates have been chosen with the origin 0 at the optical centre of the first camera. The translation vector $a$ is then identified with the point 
a at the optical centre of the second camera.

If the pair $\{R, \mathbf{a}\}$ is known then the positions $\mathbf{p}_{i}$ of the scene points relative to the two cameras are easily calculated from the image correspondences. The reconstruction problem thus reduces to the problem of recovering $\{R, a\}$ from the image correspondences. Each correspondence $q \leftrightarrow q^{\prime}$ places a single linear constraint on $\{R, \mathbf{a}\}$ of the form $(R \mathbf{q} \times R \mathbf{a}) \cdot \mathbf{q}^{\prime}=0$.

In the ambiguous case there exist at least two camera displacements, $\{R, \mathbf{a}\}$ and $\{S, \mathbf{b}\}$ compatible with the same set of image correspondences such that $\mathbf{a}$ is not parallel to $\mathbf{b}$. Let $\psi$ be the surface giving rise to the image correspondences when the relative displacement of the two cameras is $\{R, a\}$. It is shown in [9] that the equation of $\psi$ is quadratic in $\mathrm{x}$ and of the form

$$
(U \mathbf{x} \times \mathbf{x}) \cdot \mathbf{b}=(U \mathbf{a} \times \mathbf{x}) \cdot \mathbf{b}
$$

where $U=S^{\top} R$. In (2) the points $\mathbf{x}, \mathbf{a}, \mathbf{b}$ are in $\mathbf{R}^{3}$, thus $\mathbf{x}=\left[x_{1}, x_{2}, x_{3}\right]$, etc. Equation (2) for the ambiguous surface $\psi$ has the form

$$
\mathbf{x}^{\top} M \mathbf{x}+1 . \mathbf{x}=0
$$

where $M$ is a symmetric $3 \times 3$ matrix and $\mathbf{l}=U \mathbf{a} \times \mathbf{b}$. It follows from (2) and (3) that there exist vectors $\mathbf{m}$, $\mathbf{n}$ such that $\mathbf{n}$ is the axis of $U$, and such that $M$ has the form

$$
M=\frac{1}{2}(\mathbf{m} \otimes \mathbf{n}+\mathbf{n} \otimes \mathbf{m})-\mathbf{m} \cdot \mathbf{n} I
$$

Let $\theta$ be the angle of rotation of $U$. Then

$$
\mathbf{m}=\sin (\theta) \mathbf{b}+[1-\cos (\theta)] \mathbf{b} \times \mathbf{n}
$$

Equations (2) and (3) for $\psi$ are appropriate for points $\mathbf{x}$ in $\mathbf{R}^{3}$. The equations are extended to $\Pi_{\infty}$ by writing them in homogeneous form using the coordinate $x_{4}$. For example, (3) becomes

$$
\mathbf{x}^{\top} M \mathbf{x}+x_{4} \mathbf{l} \mathbf{x}=0
$$

The point $\left(x_{1}, x_{2}, x_{3}, x_{4}\right)$ is in $\psi$ if and only if it satisfies (6). The points of $\mathbf{R}^{3}$ contained in $\psi$ are unchanged because (6) reduces to (3) on setting $x_{4}=1$. The points of $\psi \cap \Pi_{\infty}$ are obtained by setting $x_{4}=0$ in (6).

The vectors $\mathbf{m}, \mathbf{n}$ of $(4)$ correspond to points $\left(m_{1}, m_{2}, m_{3}, 0\right)$ and $\left(n_{1}, n_{2}, n_{3}, 0\right)$ of $\Pi_{\infty}$. Under this correspondence, $\mathrm{m}$ and $\mathrm{n}$ are points of $\psi$ because $\mathrm{m}^{\top} M \mathrm{~m}=0$ and $\mathbf{n}^{\top} M \mathbf{n}=0$. The points $\mathrm{m}$, $\mathbf{n}$ are called the principal points of $\psi$.

A quadric surface in $\mathbf{P}^{3}$ for which the second order terms have the form $\mathbf{x}^{\top} M \mathbf{x}$ where $M$ is given by (4) is known as a rectangular quadric. Ambiguous surfaces are examples of rectangular quadrics.

\subsection{The projective geometric approach}

The projective geometric approach to reconstruction is along the following lines. Let two images of the points $p_{i}$ in space be obtained from cameras with optical centres at distinct points $o$ and $\mathbf{a}$. A point $q_{i}$ in the first image defines a projection line $\left\langle\mathbf{o}, q_{i}>\right.$ such that all points on this line project to $\mathbf{q}_{i}$, and similarly, a point $\mathbf{q}_{i}^{\prime}$ in the second image defines a projection line $\left\langle\mathbf{a}, \mathbf{q}_{i}^{\prime}\right\rangle$ such that all points on this line project to $\mathbf{q}_{i}^{\prime}$. Thus, image formation is modelled as a linear transformation from the points of $\mathbf{P}^{3}$ to the two dimensional projective space of lines (sight rays) passing through the optical centre of the camera from which the image is obtained. The lines $\left\langle\mathbf{o}, \mathbf{q}_{i}\right\rangle$ and $\left\langle\mathbf{a}, \mathbf{q}_{i}^{\prime}\right\rangle$ correspond if and only if they intersect at some point in space.

If the reconstruction is ambiguous then there exist points $\mathbf{a}, \mathbf{b}$, not both collinear with $\mathbf{o}$, such that the camera taking the second image can have its optical centre either at a or at $b$. In this case, let $q$ be the line of points projecting to a point $q$ in the first image. Let $r$ be the line of points projecting to the corresponding point $q^{\prime}$ in the second image when the optical centre of the second 
camera is at a, and let $s$ be the line of points projecting to $\mathbf{q}^{\prime}$ in the second image when the optical centre of the second camera is at $\mathrm{b}$. The line $q$ is the unique common transversal of $r$ and $s$ passing through $\mathbf{o}$, as illustrated in Figure 1.

Figure 1:

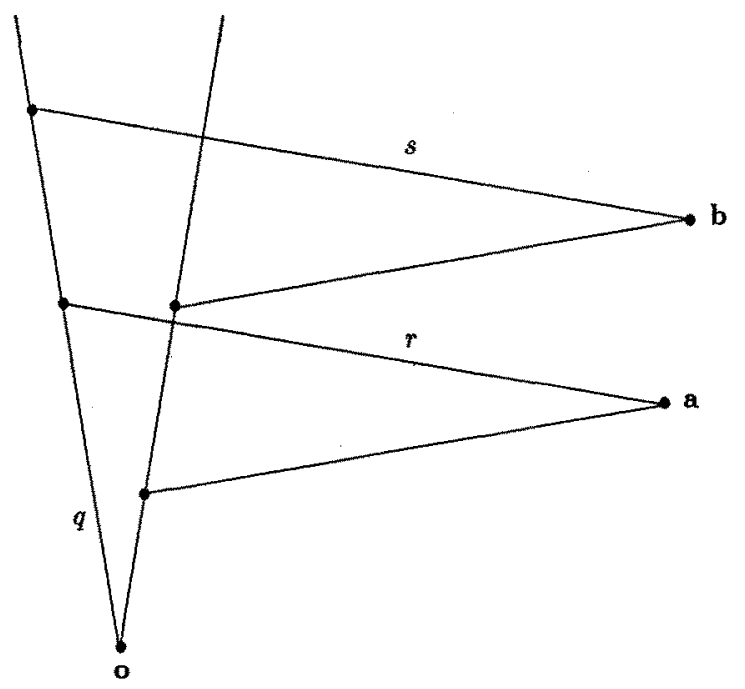

The image taken by the second camera is fixed independently of whether the camera is thought to be at $\mathbf{a}$ or $\mathbf{b}$, thus the angles between pairs of lines $r_{i}, r_{j}$ through $\mathbf{a}$ are equal to the angles between the corresponding pairs of lines $s_{i}, s_{j}$ through $\mathbf{b}$. It follows that there is a linear orthogonal (ie. angle preserving) transformation $\omega$ from the lines through a to the lines through $b$ such that $\omega r=s$.

Each line through a intersects $\Pi_{\infty}$ at a unique point, thus the space of lines through a is parameterised by $\Pi_{\infty}$. Similarly, the space of lines through $b$ is also parameterised by $\Pi_{\infty}$. If these parameterisations are adopted, then $\omega$ becomes a linear transformation from $\Pi_{\infty}$ to itself that preserves angles. Thus, $\omega$ is a rotation. In Cartesian coordinates the matrix of $\omega$ is orthogonal. In this case $\omega$ coincides with the rotation $U$ appearing in (2).

\subsection{Geometrical construction of an ambiguous surface}

The projective geometric treatment of ambiguity leads to a geometrical construction of ambiguous surfaces, as given in [17]. The transformation $\omega$ from the lines through $\mathbf{a}$ to the lines through $\mathbf{b}$ induces a linear transformation, also denoted by $\omega$, from the planes through a to the planes through b. If a line $r$ through a is contained in a plane $\Phi$, then the line $\omega r$ through $\mathbf{b}$ is contained in $\omega(\Phi)$. Let $\Pi$ be any plane containing the line $h=\omega^{-1}\langle\mathbf{o}, \mathbf{b}\rangle$. Then $\omega(\Pi)$ contains $\langle\mathbf{o}, \mathbf{b}\rangle$. As $\Pi$ varies through the one dimensional projective space of planes containing $h, \omega(I I)$ varies through the one dimensional projective space of planes containing $\langle\mathbf{o}, \mathbf{b}\rangle$. The line $l=\Pi \cap \omega(I I)$ then sweeps out the ambiguous surface, $\psi$, associated with the camera displacement from $o$ to $a$. This construction is illustrated in Figure 2.

The proof that $l$ sweeps out $\psi$ follows from the fact that, with the notation of Figure $1, q$ is the unique common transversal of $r$ and $s$. In more detail, let $l=\Pi \cap \omega(\Pi)$, as shown in Figure 2. Let $\mathbf{p}$ be any point of $l$, let $r=\langle\mathbf{a}, \mathbf{p}\rangle$, and let $s$ be the line through $\mathbf{b}$ such that $\omega r=s$. Then $s$ and $\langle\mathbf{o}, \mathbf{p}\rangle$ are contained in $\Pi$, thus $s$ intersects $\langle\mathbf{o}, \mathbf{p}\rangle$. Hence $\langle\mathbf{o}, \mathbf{p}\rangle$ is the unique common transversal of $r, s$ passing through $\mathbf{o}$. It follows that $\mathbf{p}$ is in $\psi$. The point $\mathbf{p}$ is an arbitrary point of $l$, thus $l$ is contained in $\psi$ as required. 
Figure 2:

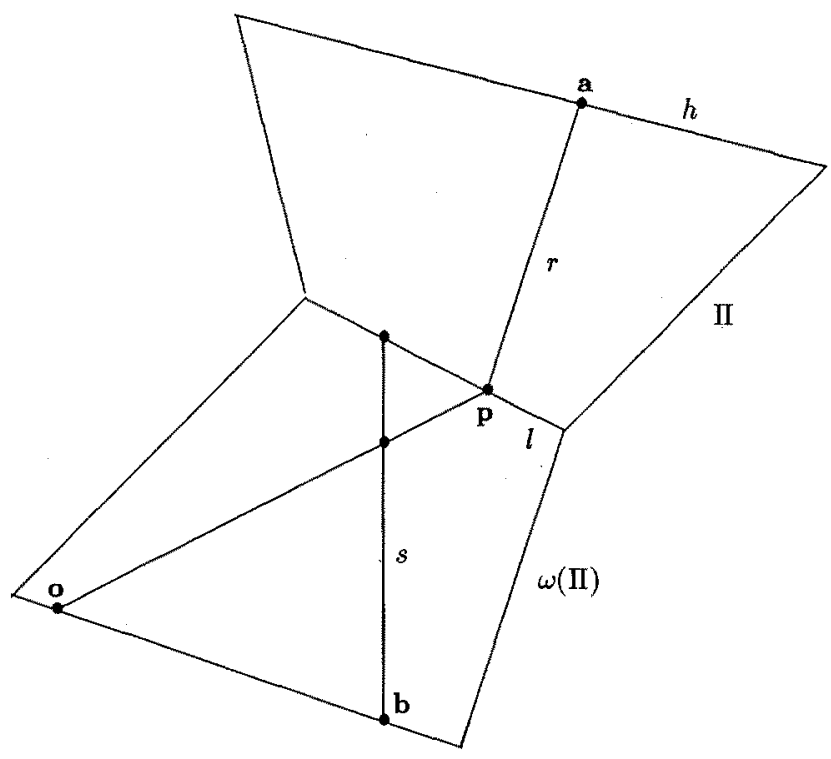

\section{Invariance of an ambiguous surface under rotation}

It is shown in Theorem 3.1 below that an ambiguous surface is invariant under a rotation through $180^{\circ}$. Before proving this result the following fact about rotations is recalled. If $\omega$ is any rotation with axis $\mathbf{n}$, and $\tau$ is a rotation through $180^{\circ}$ about an axis orthogonal to $\mathbf{n}$, then

$$
\omega=\tau \omega^{-1} \tau
$$

To prove (7) note firstly that $\omega^{-1} \tau$ reverses the direction of $\mathbf{n}$. It follows that $\omega^{-1} \tau$ is a rotation through $180^{\circ}$.

Theorem 3.1. Let $\psi$ be an ambiguous surface viewed by two cameras with optical centres at $o$ and a respectively. Let $\mathbf{b}$ be the alternative optical centre for the camera at $\mathbf{a}$. Then $\psi$ is invariant under a rotation through $180^{\circ}$ that takes a onto the line $\langle\mathbf{o}, \mathbf{b}\rangle$.

Proof. Let $\psi$ be generated by the construction described in $\S 2.3$, and let $\mathbf{m}, \mathbf{n}$ be the principal directions of $\psi$, as defined in $\S 2.1$. The collineation $\omega$ of $\$ 2.3$ is regarded as an orthogonal collineation of $\Pi_{\infty}$, with axis $\mathbf{n}$ and angle of rotation $\theta$. In this proof the point $\mathbf{b}$ serves only to fix the direction of the line $\langle\mathbf{o}, \mathbf{b}\rangle$. It is convenient to regard $\mathbf{b}$ as the point of $\Pi_{\infty}$ corresponding to the direction of $\langle\mathbf{o}, \mathbf{b}\rangle$.

Let $\tau$ be the rotation of $\Pi_{\infty}$ through $180^{\circ}$ with axis $\mathbf{m} \times \mathbf{n}$. It follows from the definition of $\tau$, on using (1), that

$$
\tau \mathbf{b}=2[\mathbf{b} .(\mathbf{m} \times \mathbf{n})] \mathbf{m} \times \mathbf{n}-\|\mathrm{m} \times \mathbf{n}\|^{2} \mathbf{b}
$$

It follows from (5) that

$$
\mathbf{m} \times \mathbf{n}=\sin (\theta) \mathbf{b} \times \mathbf{n}+[1-\cos (\theta)](\mathbf{b} \times \mathbf{n}) \times \mathbf{n}
$$

thus

$$
\text { b. }(\mathbf{m} \times \mathbf{n})=-(1-\cos (\theta))\|\mathbf{b} \times \mathbf{n}\|^{2}=-\frac{1}{2}\|\mathbf{m} \times \mathbf{n}\|^{2}
$$


Equations (8) and (9) yield

$$
\tau \mathbf{b}=\mathbf{m} \times \mathbf{n}+\mathbf{b}
$$

Now

$$
\omega^{-1} \mathbf{b}=-[1-\cos (\theta)][\mathbf{b}-(\mathbf{b} \cdot \mathbf{n}) \mathbf{n}]+\sin (\theta) \mathbf{b} \times \mathbf{n}+\mathbf{b}
$$

It follows from (10) and (11) that $\tau \mathbf{b}=\omega^{-1} \mathbf{b}$.

Let $\tau_{\psi}$ be the rotation of $\mathbf{R}^{3}$ through $180^{\circ}$ with axis in the direction $\mathbf{m} \times \mathbf{n}$ such that $\tau_{\psi}(\langle\mathbf{o}, \mathrm{b}\rangle)$ contains a. Let $h$ be the line $\omega^{-1}\langle\mathbf{o}, \mathbf{b}\rangle$, as shown in Figure 2. The restriction of $\tau_{\psi}$ to $\Pi_{\infty}$ is equal to $\tau$. Thus

$$
\tau_{\psi} \mathbf{b}=\tau \mathbf{b}=\omega^{-1} \mathbf{b}=h \cap \Pi_{\infty}
$$

It follows that $\tau_{\psi}(<\mathbf{o}, \mathbf{b}>)=h$.

Each plane $\Pi$ containing $h$ is uniquely defined by the intersection $\Pi \cap \Pi_{\infty}$. Thus, to find the planes $\omega(I)$ and $\tau_{\psi}(I I)$, it is sufficient to consider only the actions of $\omega$ and $\tau_{\psi}$ on $\Pi_{\infty}$. Let $I$ be any plane containing $\langle\mathbf{o}, \mathbf{b}\rangle$. Then $l=\Pi \cap \omega(\Pi)$ is a line in $\psi$. Let $\Xi=\tau_{\psi} \omega(\Pi)$. Then $\Xi$ contains $h$. It follows from $(7)$ that $\omega(\Xi)=\tau_{\psi} \omega^{-1} \tau_{\psi}(\Xi)$. The application of $\tau_{\psi}$ to $l$ yields

$$
\tau_{\psi}(l)=\tau_{\psi}(\Pi) \cap \tau_{\psi} \omega(\Pi)=\Xi \cap \tau_{\psi} \omega^{-1} \tau_{\psi}(\Xi)=\Xi \cap \omega(\Xi)
$$

Thus $\tau_{\psi}(l)$ is contained in $\psi$. In consequence, $\psi$ is invariant under $\tau_{\psi}$. The result that $\tau_{\psi} \mathrm{a}$ is contained in $\langle\mathbf{o}, \mathbf{b}\rangle$ follows from the construction of $\tau_{\psi}$.

It is shown in [14] that $\tau_{\psi}$ is the unique rotation through $180^{\circ}$ that leaves $\psi$ invariant and that fixes the principal points of $\psi$. A converse to Theorem 3.1 is obtained as follows.

Theorem 3,2. Let $\psi$ be a non-singular rectangular quadric and let $\tau_{\psi}$ be the rotation through $180^{\circ}$ defined in the proof of Theorem 3.1. Let $\mathbf{o}, \mathbf{a}, \tau_{\psi} \mathbf{a}$ be distinct points of $\psi$ such that $\tau_{\psi} \mathbf{a}$ is on a generator of $\psi$ passing through $\mathbf{o}$. Then $\psi$ is an ambiguous surface such that $\mathbf{o}$ is the optical centre for the camera taking the first image, and $a, \tau_{\psi}$ a are the two possible optical centres for the camera taking the second image.

Proof. Let $\mathbf{b}$ be the point at which $\left\langle\mathbf{o}, \tau_{\psi} \mathbf{a}\right\rangle$ meets $\Pi_{\infty}$. Let $\mathbf{m}, \mathbf{n}$ be the principal points of $\psi$. Let $\tau$ be the restriction of $\tau_{\psi}$ to $\Pi_{\infty}$, and let $\sigma$ be a rotation of $\Pi_{\infty}$ through $180^{\circ}$ with axis $\mathbf{b} \times \mathbf{n}$. Define the rotation $\omega$ of $\Pi_{\infty}$ by $\omega=\sigma \tau$. Then, on applying (1), $\omega^{-1} \mathbf{b}=\tau \sigma \mathbf{b}=\tau_{\psi} \mathbf{b}$.

Let $\left.h=\tau_{\psi}(<\mathbf{o}, \mathbf{b}\rangle\right)$. Then $\omega$ defines a collineation from the planes containing $h$ to the planes containing $\langle\mathbf{o}, \mathbf{b}\rangle$. Let $\psi^{\prime}$ be the ambiguous surface swept out by the lines $l=\Pi \cap \omega(\Pi)$ as $\Pi$ varies through the planes containing $h$. To prove the theorem it suffices to show that $\psi=\psi^{\prime}$.

Let $\mathbf{n}, \mathbf{i}_{n}, j_{n}$ be the fixed points of $\omega$ in $\Pi_{\infty}$. Let $k_{1}, k_{2} k_{3}$ be the unique lines containing $\mathbf{n}, \mathbf{i}_{n}$, $\mathrm{j}_{n}$, respectively, such that each $k_{i}$ meets both $h$ and $\langle\mathbf{o}, \mathbf{b}\rangle$. It follows from (2) (with $U=\omega$ ) that $\mathbf{n}, \mathbf{i}_{n}, \mathbf{j}_{n}$ are contained in $\psi$. Each $k_{i}$ meets $\psi$ at three points, thus each $k_{i}$ is contained in $\psi$. Let $\Pi=\langle h, \mathbf{n}\rangle$. Then $\omega(\Pi)=<\mathbf{o}, \mathbf{b}, \mathbf{n}\rangle$, thus

$$
\Pi \cap \omega(\Pi)=<h, \mathbf{n}>\cap<\mathbf{o}, \mathbf{b}, \mathbf{n}>=k_{1}
$$

thus $k_{1}$ is contained in $\psi^{\prime}$. Similarly, $k_{2}$ and $k_{3}$ are contained in $\psi^{\prime}$. It follows that $\psi \cap \psi^{\prime}$ contains a (split) space curve of degree five comprising $k_{1}, k_{2}, k_{3}, h,\langle\mathbf{0}, \mathrm{b}\rangle$. Two distinct quadrics intersect in a space curve of degree four only [15], thus $\psi$ and $\psi^{\prime}$ are not distinct. In other words, $\psi=\psi^{\prime}$.

An explicit expression is obtained for the rotation $\tau_{\psi}$ appearing in Theorem 3.1. The axis of $\tau_{\psi}$ has direction $\mathbf{m} \times \mathbf{n}$. Thus the action of $\tau_{\psi}$ on a general point $\mathbf{x}$ of $\mathbf{R}^{3}$ is given by

$$
\tau_{\psi} \mathbf{x}=\mathbf{s}+\frac{2[\mathbf{x} \cdot(\mathbf{m} \times \mathbf{n})] \mathbf{m} \times \mathbf{n}}{\|\mathbf{m} \times \mathbf{n}\|}-\mathbf{x}
$$

where $\mathbf{s}$ is an unknown vector normal to $\mathbf{m} \times \mathbf{n}$. It follows from (3) that the vector normal to $\psi$ at o has direction 1 . Thus the vector normal to $\psi$ at $\tau_{\psi} 0$ has direction $\tau_{\psi} l$. It follows that $2 M \mathbf{s}+1$ is 
parallel to $\tau_{\psi}$ l. Thus

$$
\mathrm{s}=-\frac{2[(\mathbf{n} . \mathbf{l}) \mathbf{m}+(\mathbf{m} . \mathbf{l}) \mathbf{n}]}{\|\mathbf{m} \times \mathbf{n}\|^{2}}
$$

It follows that

$$
\tau_{\psi} \mathbf{x}=-\frac{2[(\mathbf{n} . \mathbf{l}) \mathbf{m}+(\mathbf{m} . \mathbf{l}) \mathbf{n}]}{\|\mathbf{m} \times \mathbf{n}\|^{2}}+\frac{2[\mathbf{x} \cdot(\mathbf{m} \times \mathbf{n})] \mathbf{m} \times \mathbf{n}}{\|\mathbf{m} \times \mathbf{n}\|^{2}}-\mathbf{x}
$$

\section{Two cubic constraints on ambiguous surfaces}

Two cubic constraints on ambiguous surfaces are obtained. The first constraint is a new one arising from Theorem 3.1. The second cubic constraint is well known [5]. To obtain the first constraint the following three theorems are required.

Theorem 4.1. With the notation of Theorem 3.1, let $\psi$ be a non-singular ambiguous surface with principal points $\mathbf{m}, \mathbf{n}$, and let Cartesian coordinates be chosen such that the origin $\mathbf{o}$ is the optical centre of the camera taking the first image. Let $\mathbf{r}=\mathbf{m} \times \mathbf{n}$, let $\mathbf{l}$ be the normal to the tangent plane to $\psi$ at $\mathbf{o}$, and let a be a possible optical centre for the second camera not lying on a generator through $\mathbf{o}$. Then

$$
-4(\mathbf{m} . \mathbf{l})(\mathbf{n} . \mathbf{l})+2(\mathbf{a} . \mathbf{r})(\mathbf{l} . \mathbf{r})-(\mathbf{a} . \mathbf{l})(\mathbf{r} . \mathbf{r})=0
$$

Proof. Let $\tau_{\psi}$ be the unique non-trivial rigid involution of $\psi$ that fixes both $\mathrm{m}$ and $\mathbf{n}$. It follows from Theorem 3.1 that $\tau_{\psi} \mathbf{a}$ lies in the tangent plane to $\psi$ at $\mathbf{o}$, thus $1 . \tau_{\psi} \mathbf{a}=0$. The result follows on substituting the expression for $\tau_{\psi} \mathbf{a}$ given by (12) into the equation $1 . \tau_{\psi} \mathrm{a}=0$.

Theorem 4.2. Let $\mathrm{m}, \mathrm{n}$ be vectors, and define the matrices $N, L$ by

$$
\begin{aligned}
N & =\frac{1}{2}(\mathbf{m} \otimes \mathbf{n}+\mathbf{n} \otimes \mathbf{m}) \\
L & =(\mathbf{m} \times \mathbf{n}) \otimes(\mathbf{m} \times \mathbf{n})
\end{aligned}
$$

Let $\mathbf{e}_{1}^{\top} ; \mathbf{e}_{2}^{\top}, \mathbf{e}_{3}^{\top}$ be the rows of $N$. Then

$$
L=4\left(\begin{array}{l}
\mathbf{e}_{3}^{\top} \times \mathbf{e}_{2}^{\top} \\
\mathbf{e}_{1}^{\top} \times \mathbf{e}_{3}^{\top} \\
\mathbf{e}_{2}^{\top} \times \mathbf{e}_{1}^{\top}
\end{array}\right)
$$

Proof. The result follows from (14) and (15) on expressing $\mathbf{e}_{1}, \mathbf{e}_{2}, \mathbf{e}_{3}$ in terms of $\mathbf{m}$ and $\mathbf{n}$.

Theorem 4.3. In the reconstruction problem, let two points in space be given as optical centres for the cameras taking the first and second images respectively. Then any ambiguous surface associated with these two optical centres satisfies a cubic polynomial constraint.

Proof. Cartesian coordinates are chosen, and the notation of Theorem 3.1 is employed. Let o, a be the two optical centres, and let an ambiguous surface $\psi$ containing $\mathbf{o}$, a have an equation of the form (3). Let $\mathbf{m}, \mathbf{n}$ be the principal directions of $\psi$, and let $N$ be the matrix defined by (14). It follows from (4) and (14) that

$$
N=M-\frac{1}{2} \operatorname{Trace}(M) I
$$

The entries of $N$ are thus linear functions of the entries of $M$.

The cubic polynomial constraint on $\psi$ is obtained from (13). The term $-4(1 . m)(1 . n)$ on the left-hand side of (13) has the form

$$
-4(\mathrm{l} . \mathrm{m})(\mathrm{l} . \mathrm{n})=-4 \mathrm{I}^{\top} \mathrm{Nl}
$$

The remaining two terms on the left-hand side of (13) have the form

$$
2(\mathbf{a . r})(\mathbf{l} . \mathbf{r})-(\mathbf{a} . \mathbf{l})(\mathbf{r . r})=2 \mathbf{a}^{\top} L \mathbf{l}-(\mathbf{a} . \mathbf{l}) \operatorname{Trace}(L)
$$


It follows from (16) and (17) that (13) is equivalent to

$$
-4 \mathbf{I}^{\top} N \mathbf{l}+2 \mathbf{a}^{\top} L \mathbf{l}-(\mathbf{a} .1) \operatorname{Trace}(L)=0
$$

Let $\mathbf{e}_{1}^{\top}, \mathbf{e}_{2}^{\top}, \mathbf{e}_{3}^{\top}$ be the rows of $N$. Then, on applying Theorem 4.2, (18) takes the form

$$
-1^{\top}\left(\begin{array}{l}
\mathbf{e}_{1}^{\top} \\
\mathbf{e}_{2}^{\top} \\
\mathbf{e}_{3}^{\top}
\end{array}\right) 1+2 \mathbf{a}^{\top}\left(\begin{array}{c}
\mathbf{e}_{3}^{\top} \times \mathbf{e}_{2}^{\top} \\
\mathbf{e}_{1}^{\top} \times \mathbf{e}_{3}^{\top} \\
\mathbf{e}_{2}^{\top} \times \mathbf{e}_{1}^{\top}
\end{array}\right) 1-(\mathbf{a} .1) \operatorname{Trace}\left(\begin{array}{c}
\mathbf{e}_{3}^{\top} \times \mathbf{e}_{2}^{\top} \\
\mathbf{e}_{1}^{\top} \times \mathbf{e}_{3}^{\top} \\
\mathbf{e}_{2}^{\top} \times \mathbf{e}_{1}^{\top}
\end{array}\right)=0
$$

Equation (19) is the required cubic constraint on $\psi$.

The second, and well known, cubic polynomial constraint on the ambiguous surface $\psi$ is

$$
\operatorname{det}(N)=0
$$

where $N$ is defined by (14). Equation (20) follows from the fact that $N$ has rank two. It is cubic in the coefficients of the equation (3) defining $\psi$ because the entries of $N$ depend linearly on the entries of the matrix $M$ in (3).

\section{The case of five image correspondences}

An algebraic question arising in reconstruction is that of finding the number of essentially different camera displacements compatible with five given image correspondences. The number of unknown parameters in reconstruction is five, comprising three independent parameters for the rotation $R$, and two parameters for the direction of the translation a. Each image correspondence imposes one constraint on $\{R, \mathbf{a}\}$, thus five image correspondences are sufficient to reduce the set of compatible camera displacements to a finite size. Determining the size of this set is of interest because the number thus obtained is a fundamental algebraic measure of the complexity of the reconstruction problem.

A comment on the method of counting solutions is required. If the camera displacement $\{S, \mathbf{b}\}$ is compatible with a given set of image correspondences, then so are $\{S, \lambda \mathbf{b}\}$ and $\{\sigma S, \mathbf{b}\}$, where $\lambda$ is any non-zero scalar, and $\sigma$ is a rotation of $180^{\circ}$ with axis $S \mathbf{b}$. (See [6]). All these solutions are counted together as a single solution to the reconstruction problem. If $\{R, a\}$ is an additional camera displacement compatible with the same image correspondences as $\{S, \mathrm{~b}\}$ then the same ambiguous surface is obtained on pairing $\{R, \mathbf{a}\}$ with the $\{S, \lambda \mathbf{b}\}$ and $\{\sigma S, \mathbf{b}\}$ in turn.

Demazure [2] uses algebraic geometry to prove that the number of camera displacements compatible with five image correspondences is ten. In this context ten is high, indicating that the reconstruction problem is difficult. The following three theorems comprise a new proof of Demazure's result.

Theorem 5.1. Let five image correspondences be given compatible with a given camera displacement. Then a two dimensional space of quadrics can be constructed such that any ambiguous surface compatible with the five image correspondences and compatible with the given camera displacement is represented by a point in the two dimensional space of quadrics.

Proof. Let Cartesian coordinates be chosen with origin $o$ at the optical centre of the camera from which the first image is obtained. Let $\mathbf{q}_{i} \leftrightarrow q_{i}^{\prime}$ be five image correspondences compatible with the given camera displacement $\{R, \mathbf{a}\}$, where $R$ is an orthogonal matrix and a is the optical centre of the camera from which the second image is obtained. Let $p_{i}$ be the points in $P^{3}$ such that the image of $p_{i}$ from $o$ is $q_{i}$, and the image of $p_{i}$ from $a$ is $q_{i}^{\prime}$.

An ambiguous surface, compatible with $q_{i} \leftrightarrow q_{i}^{\prime}$ and compatible with $\{R, a\}$ contains the five points $\mathbf{p}_{i}$, together with the points $\mathbf{o}, \mathbf{a}$. The space of all quadric surfaces contained in $\mathbf{p}^{3}$ is of dimension nine. The condition that a quadric contains a known point imposes a single linear constraint on the quadric, thus the quadric surfaces containing the $\mathbf{p}_{i}, \mathbf{o}$ and $\mathbf{a}$ form $a-7=2$ 
dimensional space $\mathcal{S}^{2}$ contained in the space of all quadrics. A basis for $\mathcal{S}^{2}$ can be calculated from the $p_{i}, o$ and $a$.

Not all the quadrics parameterised by points of $\mathcal{S}^{2}$ are ambiguous surfaces. The proof that there are ten essentially different camera displacements compatible with five image correspondences relies on selecting from $\mathcal{S}^{2}$ precisely those points corresponding to ambiguous surfaces.

Theorem 5.2. Let five image correspondences be given in general position, and compatible with a known camera displacement. Then there exist two cubic plane curves $f_{1}, f_{2}$ in the space $\mathcal{S}^{2}$ constructed in Theorem 5.1 such that each camera displacement compatible with the image correspondences, but different from the known camera displacement, arises from a common zero of $f_{1}$ and $f_{2}$. Conversely, each common zero of $f_{1}$ and $f_{2}$ gives rise to essentially only one camera displacement compatible with the given image correspondences, but essentially different from the known camera displacement.

Proof. Let $\{R, \mathbf{a}\}$ be the known camera displacement and let $\left\{S_{i}, \mathbf{b}_{i}\right\}, 1 \leq i \leq n$ be the camera displacements essentially different from $\{R$, a $\}$ but also compatible with the five image correspondences. Each pair $\{R, \mathbf{a}\},\left\{S_{i}, \mathbf{b}_{i}\right\}$ yields an ambiguous surface $\psi_{i}$, with an equation of the form (2), where $\mathrm{b}=\mathbf{b}_{i}$ and $U=S_{i}^{\top} R$. Each $\psi_{i}$ corresponds to a point $\mathbf{u}_{i}$ of $\mathcal{S}^{2}$. The cubic constraints (19) and (20) yield cubic plane curves $f_{1}, f_{2}$, respectively, in $\mathcal{S}^{2}$. Each $\mathbf{u}_{i}$ is an intersection point of $f_{1}$ and $f_{2}$. Conversely, by Theorem 3.2 , each intersection point of $f_{1}$ and $f_{2}$ yields an ambiguous surface $\psi$. The surface $\psi$ yields a unique camera displacement in the set $\left\{S_{i}, \mathrm{~b}_{i}\right\}, 1 \leq i \leq n$.

Theorem 5.3. Let five image correspondences be given in general position. Then there are exactly ten essentially different camera displacements compatible with the given image correspondences.

Proof. To prove the theorem it suffices to show that the cubic plane curves $f_{1}, f_{2}$ in $\mathcal{S}^{2}$ obtained in Theorem 5.2 have, in general, exactly nine distinct intersections. The property that $f_{1}$ and $f_{2}$ have nine distinct intersections is stable against small perturbations in the coefficients of $f_{1}$ and $f_{2}$, thus it is sufficient to consider the case in which the coefficients of $f_{1}$ (defined by (19)) involving a are negligibly small in comparison with the first term $-I^{\top} N 1$. It is thus sufficient to consider the cubic curves in $\mathcal{S}^{2}$,

$$
\mathbf{l}^{\top} N \mathbf{l}=0 \quad \operatorname{det}(N)=0
$$

In order to show that the two curves of (21) have, in general, nine distinct intersections, it is sufficient to produce a single example in which they have nine intersections. To this end, choose three of the reconstructed points in $\mathbf{P}^{3}$ to be $\mathbf{p}_{1}=(1,0,0,0)^{\top}, \mathbf{p}_{2}=(0,1,0,0)^{\top}, \mathbf{p}_{3}=(0,0,1,0)^{\top}$, and choose $\mathbf{a}=(1,-1,1)^{\top}$. With these choices, the equation $\operatorname{det}(N)=0$ splits into three linear factors. It can be shown by direct calculation that $\mathbf{p}_{4}, \mathbf{p}_{5}$ can be chosen in $\mathbf{R}^{3}$ such that each line comprising $\operatorname{det}(N)=0$ meets the cubic plane curve $\mathrm{I}^{\top} N \mathrm{~N}=0$ at three distinct points.

In Theorem 5.3 the possibility is not ruled out that the intersections of the two cubic plane curves yield quadrics without real generators. Quadrics obtained in this way give rise to complex camera displacements compatible with the $q_{i} \leftrightarrow q_{i}^{\prime}$, but which are not physically acceptable.

\section{Conclusion}

The reconstruction of the relative positions of points in space from the correspondences between two different images of the points is subject to ambiguity if the points lie on certain surfaces of degree two known as rectangular hyperboloids. The ambiguous case of reconstruction has been investigated using both the projective geometric methods developed by the photogrammetrists and the Euclidean methods developed in computer vision.

A new cubic polynomial constraint on ambiguous surfaces has been obtained. This leads to a new proof of the known result that there are, in general, exactly ten camera displacements compatible with five given image correspondences. It is conjectured that these results on ambiguity are relevent to the more general problem of describing the stability of algorithms for recovering 
camera displacement from image correspondences [2]. For example, instability may arise if the two cubic plane curves obtained in Theorem 5.2 are near coincident over a wide range of points.

Acknowledgements: This work is funded by ESPRIT P2502 (Voila). The author first learnt of the German work on the reconstruction problem during an extended visit to INRIA, Rocquencourt, at the invitation of Olivier Faugeras. The author thanks Tom Buchanan for discussions on projective geometry and on the work of the photogrammetrists dating from the last century and the first half of this century. Thanks are also due to Bernard Buxton for comments on an earlier draft of this paper.

\section{References}

1. M. Demazure (1988) Sur deux problèmes de reconstruction. INRIA Rapports de Recherche No. 882.

2. J.Q. Fang and T.S. Huang (1984) Some experiments on estimating the 3-D motion parameters of a rigid body from two consecutive image frames. IEEE Transactions on Pattern Analysis and Machine Intelligence, vol. 6, pp. 545-554.

3. O.D. Faugeras and S.J. Maybank (1990) Motion from point matches: multiplicity of solutions. Accepted by Int. J. Computer Vision.

4. W. Hofmann (1950) Das Problem der "Gefärlichen Flächen" in Theorie und Praxis. Dissertation, Fakultät für Bauwesen der Technischen Hochschule München, München, FR Germany.

5. B.K.P. Horn (1987) Motion fields are hardly ever ambiguous. Int. J. of Computer Vision, vol. 1, pp. 263-278.

6. B.KP. Horn (1989) Relative Orientation. Accepted by Int. J. Computer Vision.

7. E. Kruppa (1913) Zur Ermittlung eines Objektes zwei Perspektiven mit innere Orientierung. Sitz-Ber. Akad. Wiss., Wien, math. naturw. Kl., Abt. Ila., vol 122, pp. 1939-1948.

8. H.C. Longuet-Higgins (1981) A computer algorithm for reconstructing a scene from two projections. Nature, vol. 293, pp. 133-135.

9. H.C. Longuet-Higgins (1988) Multiple interpretations of a pair of images. Proc. Roy. Soc. Lond. A, vol. 418 , pp. 1.15 .

10. H.C. Longuet-Higgins and K. Prazdny (1980) The interpretation of a moving retinal image. Proc. Roy. Soc. Lond. B, vol. 208, pp. 385-397.

11. S.J. Maybank (1985) The angular velocity associated with the optical flow field arising from motion through a rigid environment. Proc. Roy. Soc. Lond. A, vol. 401, pp. 317-326.

12. S.J. Maybank (1987) A theoretical study of optical flow. PhD Thesis, Birkbeck College, University of London.

13. S.J. Maybank (1990) Rigid velocities compatible with five image velocity vectors. Accepted by Image and Vision Computing.

14. S.J. Maybank (1990) The projective geometry of ambiguous surfaces. In preparation.

15. J.G. Semple and G.T. Kneebone (1952) Algebraic Projective Geometry. Oxford: Clarendon Press (reprinted 1979).

16. R. Sturm (1869) Das Problem der Projectivität und seine Anwendung auf die Flächen zweiten Grades. Math. Annalen, vol. 1, pp. 533-573.

17. W. Wunderlich (1942) Zur Eindeutigkeitsfrage der Hauptaufgabe der Photogrammetrie. Monatsch. Math. Physik, vol. 50, pp. 151-164. 